\title{
GLMP 160 - the first [WR] star in a binary
}

\author{
Marcin Hajduk ${ }^{1}$ Peter A. M. van Hoof ${ }^{2}$ and Albert A. Zijlstra ${ }^{3}$ \\ ${ }^{1}$ Nicolaus Copernicus Astronomical Center, ul. Rabiańska 8, 87-100 Toruń, Poland \\ ${ }^{2}$ Royal Observatory of Belgium, Ringlaan 3, 1180 Brussels, Belgium \\ ${ }^{3}$ Jodrell Bank Centre for Astrophysics, Alan Turing Building, Manchester M13 9PL, UK
}

\begin{abstract}
We discuss the results of the search for [WR] central stars in binary systems. GLMP 160 is the first [WR] central star in a binary system known. We analyze photometry, spectroscopy and imaging of this system.
\end{abstract}

Keywords. binaries: close, stars: evolution, planetary nebulae: general

\section{Introduction}

GLMP 160 (PN G222.8-04.2) was discovered as a [WR] star by Pereira \& Machado (2003). Hajduk, Zijlstra \& Gesicki (2010) have discovered GLMP 160 to be a photometric binary system. None of the $[\mathrm{WR}]$ central stars had been known to possess a close companion before, though on average about $16 \%$ of central stars of planetary nebulae are photometric binaries (Miszalski et al. 2009). [WR] stars seem to be members of binary systems less frequently than non-emission-line central stars. GLMP 160 may be important for understanding the origin and evolution of [WR] stars. We present photometric and spectroscopic observations of this object.

\section{The central star and the nebula}

GLMP 160 and its planetary nebula are shown in Fig. 1. The NTT H $\alpha$ image of the object, retrieved from the ESO archive, shows a two-component, faint planetary nebula associated with a bright emission-line star. The nebula contains two distinct components: a bipolar structure, indicated by bright wings, and a torus.

Photometry of the object was collected using the $1.0 \mathrm{~m}$ telescope at SAAO in Jan/Feb 2009, March and May 2011. We also made use of the All Sky Automated Survey (ASAS) photometric V-band catalogue (Pojmanski 2002) for GLMP 160, collected between 2001 and 2009. The mean magnitude of the star of 14.358 was close to the sensitivity limit of the survey $(V \approx 15)$. The photometric period of 0.631520 day is believed to be the result of the irradiation of the companion. The light curve seems to vary significantly between cycles (Fig. 1). Irregular changes may be due to wind activity.

Three NTT EMMI echelle spectra of the object were obtained on 2003 February 3 with the slit placed at a position angle of $-139^{\circ}, 1$ arcsec width and 10 arcsec length. Two 1800s exposures of GLMP 160 have been obtained using the HERMES spectrograph on the Mercator Telescope on 2011 February 17. Night sky lines were not subtracted from the latter spectra.

The limited diameter of the telescope allowed only few stellar lines to be detected with the HERMES spectrograph. However, variations in the line radial velocities and the evolution of the line profiles are clearly visible for the strongest lines. The spectra obtained with the Mercator telescope were taken near the photometric maximum of the system, i.e. when the irradiated hemisphere of the companion is best seen, whereas the NTT spectra were obtained near the minimum. 

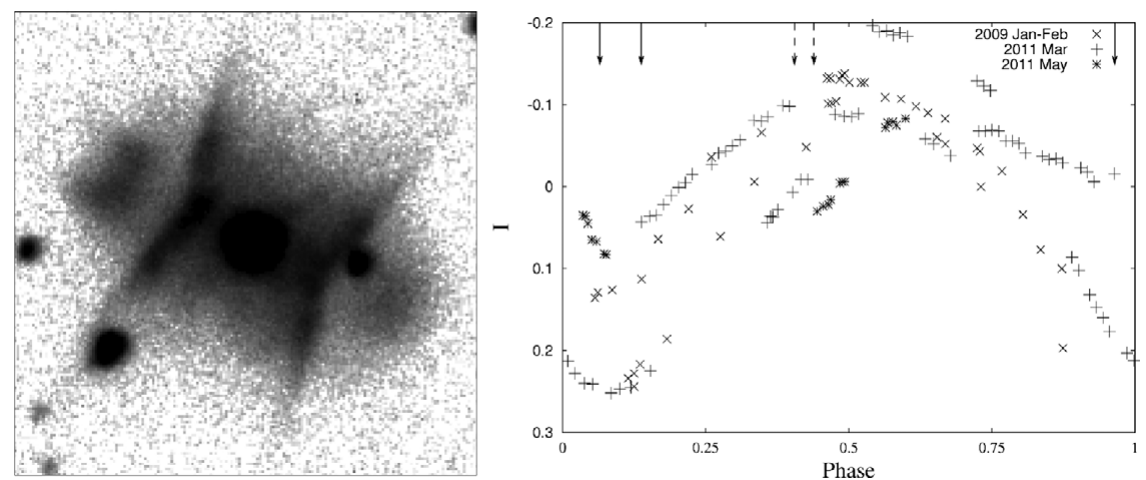

Figure 1. H $\alpha$ image of GLMP 160 (on the left). North is at the top and East to the left. The image is adopted from Hajduk, Zijlstra \& Gesicki (2010). I band photometry of GLMP 160 collected in SAAO, folded with the binary period is shown. Arrows mark the moments of the NTT (solid arrows) and Mercator telescope (dashed arrows) spectroscopic observations.

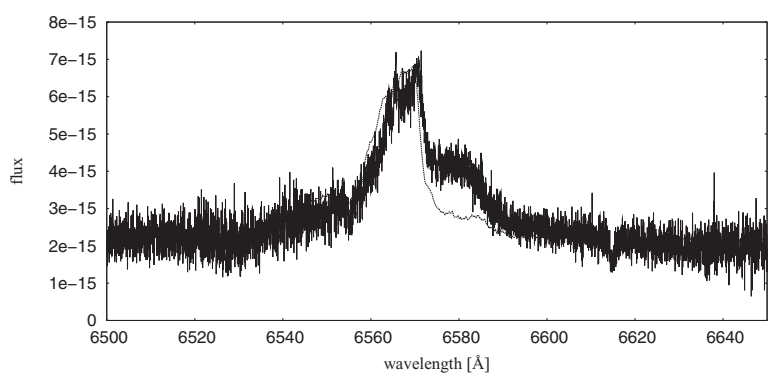

Figure 2. Spectrum of the He II $6560 \AA$ line observed with the NTT (thin) and Mercator telescope (thick). A DIB absorption is seen at $6613 \AA$. An arbitrary flux scale has been applied.

Near the photometric maximum the He II $6560 \AA$ line is redshifted with respect to the photometric minimum (Figure 2). The red component is stronger during the photometric maximum. During the photometric maximum an Na I emission component arises near the system velocity of the nebula $(+58 \mathrm{~km} / \mathrm{s})$ in addition to the interstellar absorption. We do not find an evidence for hydrogen deficiency in the nebular spectrum. The [WR] star could have been formed after the common-envelope phase. Investigation of this object may provide further constraints on the origin and evolution of the [WR] star.

\section{Acknowledgements}

We thank Peter De Cat and Patricia Lampens for obtaining the MERCATOR spectra and the discussion. This work was financially supported by MNiSW of Poland through grant No. N N203 511838. PvH acknowledges support from Belspo via the ESA PRODEX Programme.

\section{References}

Hajduk, M., Zijlstra, A. A., \& Gesicki, K. 2010, MNRAS, 406, 626

Miszalski, B., Acker, A., Moffat, A. F. J., Parker, Q. A., \& Udalski, A. 2009, A\&A, 496, 813

Pereira, C. B. \& Machado, M. 2003, A\& A, 407, 311

Pojmański, G. 2002, AcA, 52, 397 